\author{
Djacyr Magna Cabral Freire ${ }^{1}$ \\ José GuILherme CeCATtI² \\ Cláudio Sérgio Mederros Paiva ${ }^{3}$
}

\section{Artigo Original}

Palavras-chave

Útero/crescimento \& desenvolvimento Líquido amniótico

Oligodrâmnio

Polihidrâmnios

Saúde materno-infantil

Keywords

Úterus/growth \& development Amniotic fluid

Oligohydramnios

Polihydramnios

Maternal and child health

\title{
A altura uterina é capaz de diagnosticar os desvios do volume de líquido amniótico?
}

\section{Is uterine height able to diagnose amniotic fluid volume deviations?}

\section{Resumo}

OBJETIVO: Avaliar o desempenho de uma curva de altura uterina (AU) quanto à capacidade de rastrear desvios do volume de líquido amniótico, utilizando uma curva brasileira de índice de líquido amniótico (ILA) como padrão-ouro. MÉTODOS: O presente estudo representa um corte transversal no qual foram incluídas 753 gestantes em acompanhamento pré-natal na rede pública de João Pessoa (PB) no período de março a outubro de 2006 e que tiveram um exame de ultrassonografia (US) de rotina agendado para depois da $26^{a}$ semana de idade gestacional. Foram excluídos os casos com diagnóstico de gestação gemelar, óbito fetal intrauterino e malformações fetais maiores. Além de informações sociodemográficas, foram coletados também os valores da AU medida de forma padronizada, os valores do peso fetal estimado, do ILA e a idade gestacional pelo exame de US. A capacidade da curva de AU em predizer os desvios do volume de líquido amniótico foi avaliada tendo uma curva brasileira de ILA em função da idade gestacional como padrão-ouro. Para isso, foram estimados a sensibilidade, especificidade e valores preditivos positivo e negativo para diferentes pontos de corte. RESULTADOS: A medida da AU identificou 10,5\% das mulheres como AU baixa e possivelmente associada ao oligoâmnio, e 25,2\% como AU alta e possivelmente associada ao polidrâmnio. Utilizando uma curva brasileira de referência para ILA, a AU foi capaz de predizer pobremente a ocorrência de oligoâmnio (sensibilidade variando entre 37 a 28\%) e de forma razoável a ocorrência de polidrâmnio (sensibilidade variando entre 88 a 69\%). CONCLUSÃO: A medida da altura uterina mostrou um desempenho ruim para predizer oligoâmnio e um desempenho razoável para predizer polidrâmnio. Sua utilização para essa finalidade só se justifica, portanto, em situações nas quais o exame ultrassonográfico não esteja fácil e rotineiramente disponível, a fim de ajudar na priorização dos casos que deveriam ter esse exame realizado.

\section{Abstract}

PURPOSE: To evaluate the performance of a Brazilian reference curve of fundal height (FH) regarding its capacity of screening the deviations of volume of amniotic fluid using a Brazilian reference curve of amniotic fluid index (AFI) as gold standard. METHODS: This was a cross-sectional study evaluating 753 pregnant women receiving prenatal care at the public health services of João Pessoa (PB), from March to October 2006, who had a routine ultrasound exam scheduled for after 26 weeks of gestational age. Cases with diagnoses of twin pregnancy, intrauterine fetal death and major fetal malformations were excluded. Besides socio-demographic information, data regarding fundal height measured in a standard way, estimated fetal weight, AFI and gestational age at the time of the ultrasound exam were also collected. The capacity of the FH curve to predict deviations of the amniotic fluid volume was assessed using the Brazilian curve of AFI according to gestational age as the gold standard. For this purpose, sensitivity, specificity, positive and negative predictive values were estimated for different cut-off points. RESULTS: The measurement of $\mathrm{FH}$ identified $10.5 \%$ of women as having low $\mathrm{FH}$ possibly associated with oligohydramnios and $25.2 \%$ as having high $\mathrm{FH}$ possibly associated with polyhydramnios. Using a Brazilian reference curve of AFI, the $\mathrm{FH}$ was able to poorly predict the occurrence of oligohydramnios (sensitivity ranging from 37 to $28 \%$ ) and to reasonably predict the occurrence of polyhydramnios (sensitivity ranging from 88 to $69 \%$ ). CONCLUSIONS: The measurement of fundal height showed a poor performance for predicting oligohydramnios and a reasonable performance for predicting polyhydramnios. Its use for this purpose is then only supported in settings where the ultrasound exam is not easily or routinely available in order to help define priorities for cases that should have this exam performed.
Correspondêncio José Guilherme Cecatti DTG/CAISM, UNICAMP Rua Alexandre Fleming, 101 CEP: 13083-881 Campinas (SP), Brasil

Recebido

12/09/2012
Trabalho realizado no Departamento de Tocoginecologia da Faculdade de Ciências Médicas, Universidade Estadual de Campinas UNICAMP - Campinas (SP), Brasil.

'Escola Técnica em Saúde, Universidade Federal da Paraíba - UFPB - João Pessoa (PB), Brasil.

2Departamento de Tocoginecologia da Faculdade de Ciências Médicas, Universidade Estadual de Campinas - UNICAMP Campinas (SP), Brasil.

${ }^{3}$ Departamento Materno-Infantil, Universidade Federal da Paraíba - UFPB - João Pessoa (PB), Brasil.

Conflito de interesses: não há. 
Introdução

Vários estudos têm contribuído para a compreensão da regulação do volume de líquido amniótico (VLA) no decorrer da gestação ${ }^{1}$. Existem evidências estabelecendo a relação do VLA com o bem-estar fetal e apontando aumento da morbidade e mortalidade perinatal nas gestações com desvios no VLA ${ }^{2,3}$. O oligoâmnio está associado a malformações do trato urinário fetal, rotura prematura das membranas, gravidez pós-termo, insuficiência útero-placentária, restrição de crescimento fetal e parto por cesariana. O polidrâmnio está associado ao diabetes mellitus, hidropsia fetal, doença hemolítica perinatal e a malformações estruturais do feto, especialmente as obstruções altas do tubo digestivo e defeitos de fechamento do tubo neural ${ }^{4-7}$.

Conceituar desvios do VLA era um desafio antes do advento da ultrassonografia (USG) que, posteriormente, revelou-se um método revolucionário não invasivo de avaliação semiquantitativa do VLA ${ }^{8,9}$. Vários critérios têm sido propostos, como sua avaliação subjetiva ou a utilização de critérios semiquantitativos, tal como as medidas vertical, bidimensional (diâmetro vertical+diâmetro horizontal) do maior bolsão de LA e o índice de líquido amniótico (ILA) obtido pela técnica dos quatro quadrantes?.

O ILA é o método mais utilizado atualmente por apresentar pequena variabilidade interobservador ${ }^{10}$, embora existam controvérsias sobre sua capacidade em predizer o VLA $^{11}$. Moore e Cayle ${ }^{12}$ definiram oligoâmnio quando o ILA estava abaixo do percentil 5 , e polidrâmnio quando acima do percentil 95 . Rutherford et al. ${ }^{13}$ propuseram um ILA de $5 \mathrm{~cm}$ como limiar semiquantitativo para classificar oligoâmnio. Já para Jeng et al. ${ }^{14}$, o melhor ponto de corte foi $8 \mathrm{~cm}$, pois verificaram que gestações com ILA abaixo desse valor apresentaram maior incidência de líquido amniótico meconial, de cesariana por sofrimento fetal agudo, de cardiotocografia anormal e de escore de Apgar menor que 7 no primeiro minuto.

Além da indefinição sobre os pontos de corte, um aspecto importante a se considerar é qual o padrão de referência a ser adotado. Embora existam algumas curvas de normalidade do ILA construídas com populações brasileiras ${ }^{15,16}$, ainda são utilizadas para gestantes brasileiras curvas de ILA referentes a países desenvolvidos ou a populações com características étnicas e sociais distintas ${ }^{3}$.

Por outro lado, no contexto da assistência pré-natal, é importante considerar quais critérios clínicos indicam a necessidade de avaliação do VLA, já que o ILA parece ser um valioso marcador ultrassonográfico de diversos processos patológicos fetais ou maternos. A medida seriada da altura uterina (AU) no decorrer da gestação representa o principal recurso clínico para avaliar o crescimento fetal, como também para identificar outras complicações na gravidez, como gemelidade, mola hidatiforme, macrossomia fetal, polidrâmnio e oligoâmnio. As medidas da AU também podem ser usadas para identificação de erros na estimativa da idade da gestação (IG) baseada na data da última menstruação (DUM). Trata-se de um recurso clínico útil e com baixo custo, porém não adequadamente valorizado na rotina de pré-natal.

Sabe-se que a medida da AU é resultante da interação de múltiplas variáveis, sendo fortemente correlacionada ao peso fetal e ao volume do líquido amniótico, e que também é frequente a associação entre desvio do crescimento e alteração do VLA. Além disso, muitos casos de restrição do crescimento fetal (RCF) cursam com oligoâmnio, e muitos casos de macrossomia fetal com polidrâmnio. Assim, a utilização durante a assistência pré-natal de curvas de altura uterina em função da idade gestacional para rastrear desvios de crescimento fetal poderia contribuir, também, para rastrear desvios do volume de líquido amniótico. E ainda, por ser procedimento simples, não invasivo e de baixo custo, a medida da AU pode assumir maior importância em países em desenvolvimento, onde recursos tecnológicos mais sofisticados para avaliar a saúde fetal podem não estar disponíveis de rotina ou mesmo estar ausentes.

No Brasil, a curva de normalidade de crescimento da altura uterina para a idade gestacional padronizada pelo Ministério da Saúde é uma curva de origem uruguaia, sendo considerados limites de normalidade para o crescimento uterino, respectivamente, o percentil 10 para o limite inferior e o percentil 90, para o limite superior ${ }^{17}$. São poucos os estudos que relacionam uma curva de crescimento do útero com a idade gestacional no país ${ }^{18,19}$.

$\mathrm{Na}$ atenção à gravidez de alto risco a USG já é rotineiramente indicada, porém na atenção à gravidez de baixo risco os critérios clínicos para indicar a realização de USG ainda não estão claramente definidos, ou não foram suficientemente avaliados quanto ao seu desempenho em rastrear os desvios do VLA, ou ainda não estão disponíveis na prática para uma parcela importante das mulheres que engravidam e buscam atenção pré-natal em unidades básicas de saúde. Empiricamente, a medida da AU tem sido referida como um parâmetro para identificar gestantes com suspeita de polidrâmnio ou oligoâmnio; porém, não se encontram referências ao desempenho de curvas de AU de populações brasileiras quanto à capacidade de rastrear desvios do VLA.

Por esse motivo, o objetivo deste estudo foi o de avaliar o desempenho de uma curva brasileira de altura uterina quanto à capacidade de rastrear desvios do volume de líquido amniótico (oligoâmnio ou polidrâmnio) utilizando uma curva brasileira de ILA como padrão-ouro. 
Métodos

Esta é uma análise secundária do estudo originalmente realizado para validar a curva de referência de altura uterina por idade gestacional de Freire et al. ${ }^{18}$ em sua capacidade de predizer os desvios do crescimento fetal, e detalhes adicionais sobre o método empregado podem ser encontrados na publicação original ${ }^{20}$. Os dados referentes a 753 gestantes que foram acompanhadas durante o pré-natal em um hospital universitário e em uma maternidade pública da cidade de João Pessoa (PB) foram incluídos na presente análise. As gestantes foram originalmente incluídas no estudo pelo Setor de Ultrassonografia de cada uma das instituições participantes, quando tinham agendado um exame de rotina a partir da $26^{a}$ semana de gestação, no período de março a outubro de 2006. O cálculo do tamanho amostral para o estudo original foi realizado com base a uma estimativa de prevalência de restrição de crescimento fetal para essa população de $12 \%$, resultando em indicação para inclusão de 750 mulheres. Como a prevalência estimada de alterações do VLA é aproximadamente a mesma, esse número foi considerado suficiente para a presente análise de validação da curva em sua capacidade de predizer os desvios do VLA, utilizando como padrão-ouro a curva de valores de referência do ILA para a idade gestacional de Machado et al. ${ }^{15}$.

Foram incluídas no estudo as mulheres que tinham uma IG de 26 semanas ou mais (calculada pela data da última menstruação conhecida ou confirmada por um exame de ultrassonografia (US) realizado na primeira metade da gestação), independentemente de sua condição de risco. Foram excluídas as que apresentavam gestação gemelar, óbito fetal ou malformações fetais. Para cada mulher foram coletadas informações sobre as seguintes variáveis: idade, índice de massa corporal (IMC, classificado conforme os valores de referência por idade gestacional de Atalah recomendados pelo Ministério da Saúde ${ }^{17}$ ), cor da pele, paridade, número de cesáreas anteriores, hábito de fumar. Foram também coletadas outras variáveis principais de interesse sobre medida da AU (de forma padronizada conforme recomendação ${ }^{20}$ ), peso fetal estimado pelo US e índice de líquido amniótico (ILA) medido pela técnica dos quatro quadrantes ${ }^{9}$ e classificado posteriormente em oligoâmnio (abaixo do percentil 10), normoâmnio (entre percentil 10 e 90) e polidrâmnio (acima do percentil 90), utilizando a curva brasileira de valores de referência de ILA por idade gestacional de Machado et al. ${ }^{15}$ como padrão-ouro.

As medidas de AU foram realizadas nas gestantes frequentando as consultas de pré-natal e o US, rotineiramente solicitado. Os dados foram originalmente inseridos em uma ficha padrão pelo examinador. Os exames de ultrassom foram realizados com os equipamentos GE Voluson 730 PRO e GE Logic 500, ambos utilizando transdutores convexos multifrequenciais $(2,5-5,0 \mathrm{MHz})$, por examinadores treinados e experientes. $\mathrm{O}$ estudo foi previamente aprovado pelo Comitê de Ética em Pesquisa de ambas as instituições onde as mulheres foram selecionadas. A participação das mulheres era voluntária e sua inclusão no trabalho só ocorria após terem sido apresentadas ao estudo, terem suas dúvidas respondidas pelo pesquisador e o correspondente termo de consentimento livre e esclarecido devidamente lido e assinado.

Para a abordagem analítica, inicialmente são descritas as distribuições percentuais das mulheres participantes entre as categorias das variáveis sociodemográficas e de antecedentes obstétricos. Os valores do ILA para cada mulher do estudo foram plotados no gráfico da curva de valores de referência de ILA em função da IG ${ }^{15}$, utilizando os três possíveis pontos de corte para a determinação tanto do volume de líquido amniótico diminuído - oligoâmnio (ILA abaixo dos percentis 2,5 ou 5 ou 10), quanto do volume de líquido amniótico aumentado — polidrâmnio (ILA acima dos percentis 97,5 ou 95 ou 90). Em seguida, os valores de AU considerados diminuídos (abaixo do percentil 10) ou aumentados (acima do percentil 90) pela curva de valores de referência de altura uterina por IG de Freire et al. ${ }^{18}$ foram validados como capazes de predizer o diagnóstico de oligoâmnio e polidrâmnio (por três diferentes pontos de corte), segundo a curva brasileira de ILA em função da IG de Machado et al. ${ }^{15}$ Para isso foi avaliado o desempenho da AU calculando-se a sensibilidade, especificidade e valores preditivos positivo e negativo para cada ponto de corte. Para esses procedimentos foi utilizado o software SPSS.

\section{Resultados}

Foram incluídas no estudo 753 mulheres com idade variando de 14 a 45 anos, com média de 26,5 anos. O fenótipo racial predominante foi a cor branca $(46,6 \%)$, o índice de massa corporal das mulheres estudadas variou entre 17,8 a $45,5 \mathrm{~kg} / \mathrm{m}^{2}$, com média $27 \mathrm{de} \mathrm{kg} / \mathrm{m}^{2}$ e proporção elevada de quase $30 \%$ de sobrepeso e obesidade. Quanto ao hábito de fumar, a maioria não era tabagista $(97,6 \%)$. A Tabela 1 mostra que, com relação às características obstétricas, metade das gestantes eram nulíparas e 19,2\% tinham antecedentes de cesárea. A IG variou de 29 a 41 semanas e a grande maioria $(80,1 \%)$ foi incluída com mais de 32 semanas.

Pela medida da altura uterina foram diagnosticados 79 casos abaixo do percentil 10, o que corresponde a $10,5 \%$ do total de casos com AU diminuída, 484 entre o percentil 10 e $90(64,3 \%)$ e 190 casos acima do percentil 90 (25,2\%), com AU elevada. A Figura 1 apresenta a dispersão das medidas do ILA por IG pelos pontos de corte (percentis 2,5 e 97,5; 5 e 95; 10 e 90) 
da curva de ILA por idade gestacional. Dos 753 casos, $116(15,4 \%)$ foram classificados abaixo do percentil 10, o que corresponde a oligoâmnio; $575(76,4 \%)$ entre o percentil 10 e 90 , e $62(8,2 \%)$ acima do percentil 90 , o que corresponde a polidrâmnio.

Tabela 1. Distribuição percentual das gestantes segundo algumas características demográficas e obstétricas

\begin{tabular}{|c|c|c|}
\hline Características & n & $\%$ \\
\hline \multicolumn{3}{|l|}{ Idade (anos) } \\
\hline $14-19$ & 105 & 13,9 \\
\hline $20-29$ & 405 & 53,8 \\
\hline$\geq 30$ & 243 & 32,3 \\
\hline \multicolumn{3}{|c|}{ Fenótipo racial predominante } \\
\hline Branca & 351 & 46,6 \\
\hline Parda & 341 & 45,3 \\
\hline Negra & 61 & 8,1 \\
\hline \multicolumn{3}{|c|}{ Índice de massa corporal (IMC) } \\
\hline Baixo peso & 142 & 18,9 \\
\hline Adequado & 390 & 51,8 \\
\hline Sobrepeso & 162 & 21,5 \\
\hline Obesidade & 59 & 7,8 \\
\hline \multicolumn{3}{|l|}{ Fumo } \\
\hline Sim & 18 & 2,4 \\
\hline Não & 735 & 97,6 \\
\hline \multicolumn{3}{|l|}{ Paridade } \\
\hline 0 & 380 & 50,5 \\
\hline $1-2$ & 348 & 46,2 \\
\hline$\geq 3$ & 25 & 3,3 \\
\hline \multicolumn{3}{|c|}{ Antecedente de cesárea } \\
\hline Sim & 145 & 19,3 \\
\hline Não & 608 & 80,7 \\
\hline \multicolumn{3}{|c|}{ Idade gestacional } \\
\hline $26-36$ & 432 & 57,4 \\
\hline$\geq 37$ & 321 & 42,6 \\
\hline Total & 753 & 100,0 \\
\hline
\end{tabular}

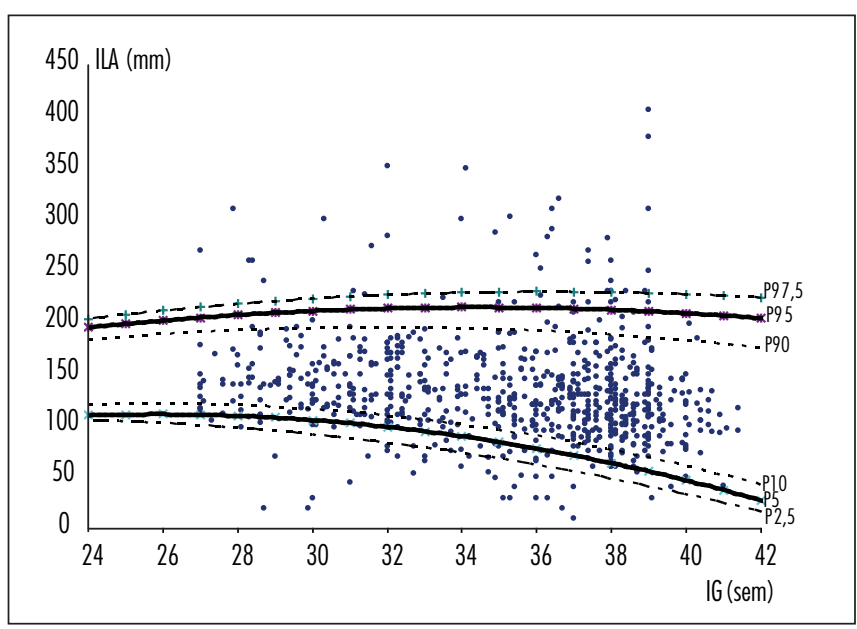

Figura 1. Distribuição das 753 medidas do índice de líquido amniótico pelos pontos de corte (percentis 2,$5 ; 5 ; 10 ; 90 ; 95$ e 97,5) da curva de Machado et al. ${ }^{15}$.
O desempenho da curva de altura uterina para o diagnóstico de oligoâmnio tendo a curva de índice de líquido amniótico como padrão-ouro está sumarizado na Tabela 2, e para diagnóstico de polidrâmnio na Tabela 3. Quando se compara o desempenho da curva de altura uterina para o diagnóstico de oligoâmnio nos três pontos de corte da curva de ILA (percentis 2,5, 5 e 10, respectivamente) observa-se que a sensibilidade foi baixa nos três pontos de corte, porém diminuiu de 37,5 para 34,8 e $28,5 \%$ em função dos pontos de corte, nessa ordem. Por outro lado, a especificidade foi alta nos três pontos de corte, tendo apresentado um pequeno aumento em função dos pontos de corte. Já para o diagnóstico de polidrâmnio, a sensibilidade foi melhor para os três pontos de corte (percentis 97,5, 95 e 90, respectivamente), diminuindo também em função dos pontos de corte de 88,6 para 76,1 e $69,4 \%$, enquanto a especificidade praticamente não variou, ficando em torno de $78 \%$.

Tabela 2. Sensibilidade, especificidade, valores preditivos positivo e negativo da medida da altura uterina para detecção de oligoâmnio, considerando diferentes pontos de corte da curva de normalidade do índice de líquido aminiótico de Machado et al. ${ }^{15}$ como padrão-ouro ( $\mathrm{n}=753$ )

\begin{tabular}{|c|c|c|c|c|c|c|}
\hline \multirow{2}{*}{ 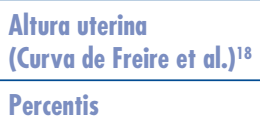 } & \multicolumn{6}{|c|}{$\begin{array}{l}\text { Índice de líquido amniótico } \\
(\text { Curva de Machado et al.o) }\end{array}$} \\
\hline & $\leq P 2,5$ & $>P 2,5$ & $\leq P 5$ & $>P 5$ & $\leq P 10$ & $>P 10$ \\
\hline$\leq \mathrm{P} 10$ & 12 & 67 & 24 & 55 & 33 & 46 \\
\hline$>\mathrm{P} 10$ & 20 & 654 & 45 & 629 & 83 & 591 \\
\hline \multirow[t]{3}{*}{ Total } & 32 & 721 & 69 & 684 & 116 & 637 \\
\hline & \multicolumn{6}{|c|}{ Desempenho (\%) } \\
\hline & \multicolumn{2}{|c|}{ Percentil 2,5 } & \multicolumn{2}{|c|}{ Percentil 5} & \multicolumn{2}{|c|}{ Percentil 10} \\
\hline Sensibilidade & \multicolumn{2}{|c|}{37,5} & \multicolumn{2}{|c|}{34,8} & \multicolumn{2}{|c|}{28,5} \\
\hline Especificidade & \multicolumn{2}{|c|}{90,7} & \multicolumn{2}{|c|}{92,0} & \multicolumn{2}{|c|}{92,8} \\
\hline Valor preditivo positivo & \multicolumn{2}{|c|}{15,2} & \multicolumn{2}{|c|}{30,4} & \multicolumn{2}{|c|}{41,8} \\
\hline Valor preditivo negativo & \multicolumn{2}{|c|}{97,0} & \multicolumn{2}{|c|}{93,3} & \multicolumn{2}{|c|}{877} \\
\hline
\end{tabular}

Tabela 3. Sensibilidade, especificidade, valores preditivos positivo e negativo da medida da altura uterina para detecção de polidrâmnio, considerando diferentes pontos de corte da curva de normalidade do índice de líquido amniótico de Machado et al. ${ }^{15}$ como padrão ouro ( $n=753)$

\begin{tabular}{|c|c|c|c|c|c|c|}
\hline \multirow{2}{*}{$\begin{array}{l}\text { Altura uterina } \\
\text { (Curva de Freire ef al., 2006) } \\
\text { Percentis }\end{array}$} & \multicolumn{6}{|c|}{$\begin{array}{l}\text { Índice de líquido amniótico } \\
\text { (Curva de Machado ef alo, 2007) }\end{array}$} \\
\hline & $\geq P 97,5$ & $<P 97,5$ & $\geq P 95$ & $<P 95$ & $\geq P 90$ & $<P 90$ \\
\hline$\geq P 90$ & 31 & 159 & 35 & 155 & 43 & 147 \\
\hline$<P 90$ & 4 & 559 & 11 & 552 & 19 & 544 \\
\hline \multirow[t]{3}{*}{ Total } & 35 & 718 & 46 & 707 & 62 & 691 \\
\hline & \multicolumn{6}{|c|}{ Desempenho (\%) } \\
\hline & \multicolumn{2}{|c|}{ Percentil 97,5 } & \multicolumn{2}{|c|}{ Percentil 95} & \multicolumn{2}{|c|}{ Percentil 90} \\
\hline Sensibilidade & \multicolumn{2}{|c|}{88,6} & \multicolumn{2}{|c|}{76,1} & \multicolumn{2}{|c|}{69,4} \\
\hline Especificidade & \multicolumn{2}{|c|}{77,9} & \multicolumn{2}{|c|}{78,1} & \multicolumn{2}{|c|}{78,7} \\
\hline Valor preditivo positivo & \multicolumn{2}{|c|}{16,3} & \multicolumn{2}{|c|}{18,4} & \multicolumn{2}{|c|}{22,6} \\
\hline Valor preditivo negativo & \multicolumn{2}{|c|}{98,2} & \multicolumn{2}{|c|}{98,1} & \multicolumn{2}{|c|}{96,6} \\
\hline
\end{tabular}




\section{Discussão}

Este estudo teve como objetivo avaliar o desempenho da medida da altura uterina no rastreamento de desvios do volume de líquido amniótico em uma população heterogênea de gestantes, dentro de condições de aplicação prática similares à realidade dos serviços públicos de saúde no Brasil. Como a amostra do estudo foi selecionada de acordo com a livre demanda de dois serviços públicos, as características demográficas e obstétricas das gestantes se assemelham às de outros estudos na população brasileira para obtenção de curvas de AU e de ILA. Chama a atenção o fato de que quase um terço da amostra tenha sido classificada como portadora de sobrepeso ou obesidade pelo índice de massa corporal, especialmente em uma população de baixa renda no nordeste do Brasil. Isso pode influenciar a medida da AU e, consequentemente, alterar a avaliação do VLA. Essa característica amostral pode estar relacionada à distribuição do IMC na população, pois se verificou uma associação significativa entre IMC e medida da AU. Entre as gestantes classificadas como acima do P90 na curva de AU, a proporção classificada pelo IMC pelos critérios recomendados pelo Ministério da Saúde ${ }^{17}$ como sobrepeso ou obesidade $(46,3 \%)$ foi significativamente maior que entre as abaixo do P90 na curva de $\mathrm{AU}(23,1 \%)$ $\left(\chi^{2}=36 ; p<0,0001\right.$; dados não apresentados em tabela).

É importante ressaltar que este estudo utilizou como padrão-ouro a curva de ILA por IG construída com um grupo de mulheres brasileiras ${ }^{15}$, para avaliar a capacidade diagnóstica de oligoâmnio e polidrâmnio pela AU. A opção deste estudo por não utilizar a curva de Moore e Cayle ${ }^{12}$ como padrão-ouro - o que frequentemente acontece com os estudos que utilizam curvas de ILA — deveu-se ao fato de que uma curva de ILA, construída com dados de uma amostra da população brasileira, provavelmente seria mais adequada para populações de países em desenvolvimento, e que essa hipótese deveria ser averiguada.

Este estudo também parece ser o primeiro que teve como objetivo correlacionar a medida da AU com os desvios do VLA. Em uma revisão feita em várias bases bibliográficas sobre o tema, abrangendo os últimos 20 anos, não foram encontradas referências de estudos assim realizados. Por outro lado, são inúmeras as referências ao uso da medida da AU como método clínico para detectar fetos pequenos ou grandes para a idade gestacional. Há uma ampla variação no desempenho da medida da $\mathrm{AU}$ como método de rastreamento dos desvios de crescimento fetal. A sensibilidade para detectar fetos PIG tem variado de 26,4 a 86\%. Vários estudos têm mostrado discrepância entre as curvas de AU quando aplicadas a outras populações diferentes das que foram parâmetros para sua construção. Como não foram identificados estudos semelhantes a este, não foi possível comparar os resultados. Mesmo assim, supõe-se que a variação do desempenho diagnóstico entre as curvas de crescimento de AU seja influenciada não apenas por características sociodemográficas, mas também por fatores de ordem metodológica entre os estudos, pela padronização da técnica de medida da AU utilizada, população estudada (gestantes de alto ou de baixo risco), e principalmente pelos critérios ultrassonográficos de definição de oligoâmnio e polidrâmnio pelo ILA.

Nessa mesma casuística, Freire et al..$^{20}$ encontraram uma sensibilidade de 51,6\% da curva de AU em detectar PIG pelo peso fetal estimado por ultrassonografia (PFE) e sensibilidade de $85,7 \%$ para detectar PIG pelo peso neonatal, bem superior à sensibilidade da curva adotado pelo Ministério da Saúde (12,5 e 42,9\%). Concluíram que esses resultados já são suficientes para recomendar seu uso na prática clínica do rastreamento pré-natal de desvios de crescimento fetal.

Neste estudo, o desempenho da curva de AU para o diagnóstico de oligoâmnio tendo a curva de ILA como padrão-ouro ficou muito abaixo do esperado para um bom método de rastreamento. Quando se compara o desempenho da curva de AU nos três pontos de corte da curva de ILA, observa-se que a sensibilidade diminuiu em função do aumento dos pontos de corte. Por outro lado, a especificidade foi alta nos três pontos de corte, tendo apresentado um pequeno aumento em função dos pontos de corte. Já para o diagnóstico de polidrâmnio, a sensibilidade foi melhor para os três pontos de corte, diminuindo também em função do aumento dos pontos de corte, enquanto a especificidade praticamente não variou. Neste estudo optou-se por calcular o desempenho diagnóstico em três pontos de corte devido à falta de consenso na literatura sobre os percentis limiares da curva de normalidade do ILA $^{3,10}$, embora se perceba uma tendência em se utilizar os percentis 2,5 e 97,5 ou percentis 5 e $95^{12,16}$.

Mesmo considerando que uma sensibilidade de $37,5 \%$ para detectar oligoâmnio e de $88,6 \%$ para detectar polidrâmnio está aquém do desejado para um bom método de rastreamento, é válido supor, então, que a curva de AU de Freire et al. ${ }^{18}$ provavelmente também apresenta melhor desempenho para essa finalidade do que a curva recomendada pelo Ministério da Saúde. É importante ressaltar que o objetivo deste estudo foi validar a curva de AU para rastreamento dos desvios do VLA no contexto da prática clínica, especialmente visando a sua utilização na assistência pré-natal em nível de atenção básica. Como não são ainda bem definidos os critérios clínicos que indicam a necessidade de avaliação do VLA em gestantes de baixo risco, e que o ILA parece ser um valioso marcador ultrassonográfico de diversos processos patológicos fetais ou maternos ${ }^{21}$, a medida da AU se apresenta como um critério clínico objetivo, facilmente mensurável e factível em todos os níveis de assistência pré-natal. 
Esses dados apontam a necessidade da valorização de métodos de rastreamento viáveis e adequados à realidade brasileira no contexto da assistência à saúde materno-infantil. Assim, a utilização durante a assistência pré-natal de curvas de altura uterina em função da idade gestacional para rastrear também desvios do volume de líquido amniótico, além de rastrear desvios do crescimento fetal, pode contribuir ainda para reduzir a morbidade e mortalidade fetal. É evidente que a comprovação de qualquer suspeita só poderá ter uma avaliação mais detalhada com um exame de ultrassonografia, mas a medida da altura uterina pode ser uma ferramenta de ajuda no estabelecimento de prioridades sobre quem e quando deve ter o exame de imagem realizado.

\section{Referências}

1. Beall MH, van den Wijngaard JP, van Gemert M, Ross MG. Regulation of amniotic fluid volume. Placenta. 2007;28(8-9):824-32.

2. Moore TR. The role of amniotic fluid assessment in evaluating fetal well-being. Clin Perinatol. $2011 ; 38(1): 33-46$.

3. Machado MR, Cecatti JG, Santos FF, Marussi EF, Parpinelli MA. [Variability of amniotic fluid volume with gestational age according to some sociodemographic and obstetrical variables among lowrisk pregnancies]. Rev Bras Ginecol Obstet. 2003;25(9):639-46. Portuguese.

4. Magann EF, Chauhan SP, Doherty DA, Lutgendorf MA, Magann MI, Morrison JC. A review of idiopathic hydramnios and pregnancy outcomes. Obstet Gynecol Surv. 2007;62(12):795-802.

5. Visvalingam G, Purandare N, Cooley S, Roopnarinesingh R, Geary M. Perinatal outcome after ultrasound diagnosis of anhydramnios at term. J Obstet Gynaecol. 2012;32(1):50-3.

6. Leibovitch L, Kuint J, Rosenfeld E, Schushan-Eisen I, WeissmannBrenner A, Maayan-Metzger A. Short-term outcome among term singleton infants with intrapartum oligohydramnios. Acta Paediatr. 2012;101(7):727-30.

7. Morris JM, Thompson K, Smithey J, Gaffney G, Cooke I, Chamberlain $P$, et al. The usefulness of ultrasound assessment of amniotic fluid in predicting adverse outcome in prolonged pregnancy: a prospective blinded observational study. BJOG. 2003;110(1 1):989-94.

8. Manning FA, Hill LM, Platt LD. Qualitative amniotic fluid volume determination by ultrasound: antepartum detection of intrauterine growth retardation. Am J Obstet Gynecol. 1981;139(3):254-8.

9. Phelan JP, Smith CV, Broussard P, Small M. Amniotic fluid volume assessment with the four-quadrant technique at 36-42 weeks' gestation. J Reprod Med. 1987;32(7):540-2.

10. Chang TC, Yeo SH, Huang HF, Leng JH. Reproducibility of the amniotic fluid index: its effect on clinical practice. Ultrasound Obstet Gynecol. 1995;6(6):416-20.

11. Magann EF, Sandlin AT, Ounpraseuth ST. Amniotic fluid and the clinical relevance of the sonographically estimated amniotic fluid volume: oligohydramnios. J Ultrasound Med. $2011 ; 30(11): 1573-85$.

12. Moore TR, Cayle JE. The amniotic fluid index in normal human pregnancy. Am J Obstet Gynecol. 1990; 162(5): 1 168-73.

13. Rutherford SE, Phelan JP, Smith CV, Jacobs N. The four-quadrant assessment of amniotic fluid volume: an adjunct to antepartum fetal heart rate testing. Obstet Gynecol. 1987;70(3 Pt 1):353-6.

14. Jeng CJ, Lee JF, Wang KG, Yang YC, Lan CC. Decreased amniotic fluid index in term pregnancy. Clinical significance. J Reprod Med. 1992;37(9):789-92.

15. Machado MR, Cecatti JG, Krupa F, Faundes A. Curve of amniotic fluid index measurements in low-risk pregnancy. Acta Obstet Gynecol Scand. 2007;86(1):37-41.

16. Velho MT, Morais EN, Ethur AB. [Ultrasonographic assessment of the amniotic fluid index in normal pregnancies from the 12th to the 42nd week of pregnancy]. Rev Bras Ginecol Obstet. $2001 ; 23(4)$ :225-32. Portuguese.

17. Brasil. Ministério da Saúde. Secretaria de Atenção à Saúde. Departamento de Ações Programáticas Estratégicas. Área Técnica de Saúde da Mulher. Pré-natal e puerpério: atenção qualificada e humanizada. Manual técnico. Brasília (DF): Ministério da Saúde; 2005.

18. Freire DM, Paiva CS, Coelho EA, Cecatti JG. [Curve of fundal height measurements according to gestation age among low-risk pregnant women]. Rev Bras Ginecol Obstet. 2006;28(1):3-9. Portuguese.

19. Martinelli S, Bittar RE, Zugaib M. [Proposal of a new uterine height growth curve for pregnancies between 20 and 42 weeks]. Rev Bras Ginecol Obstet. 2001;23(4):235-41. Portuguese.

20. Freire DM, Cecatti JG, Paiva CS. Symphysis-fundal height curve in the diagnosis of fetal growth deviations. Rev Saúde Pública. 2010;44(6):1031-8.

21. Ross MG. Value of the amniotic fluid index compared with the single deepest pocket. Obstet Gynecol. 2005; 105(2):439-40. 